\title{
КОНДУКТИВНЫЙ ТЕПЛОПЕРЕНОС В СЛОЕ ТОНКОПЛЕНОЧНОЙ ТЕПЛОВОЙ ИЗОЛЯЦИИ
}

\author{
Половников Вячеслав Юрьевич, \\ polov@tpu.ru \\ Национальный исследовательский Томский политехнический университет, \\ Россия, 634050, г. Томск, пр. Ленина, 30.
}

\begin{abstract}
Актуальность исследования обусловлена необходимостью разработки новых энергосберегающих технологий для тепловой защиты элементов систем теплоснабжения и иного энергетического оборудования различного назначения. Высокий уровень тепловых потерь в системах теплоснабжения (например, тепловые сети, ТЭС, котельные) и энергетическом оборудовании различного назначения (химические производства, пищевая промышленность и т. Д.) и неудовлетворительное состояние их тепловой изоляции обосновывают необходимость разработки новых технологий снижения уровня тепловых потерь в рассматриваемых системах. Уникальные теплофизические характеристики тонкопленочных теплоизоляционных покрытий позволяют использовать их в различных энергетических системах и оборудовании. Несмотря на это, технологии применения тонкопленочных теплоизоляционных покрытий к настоящему моменту времени не получили развития. Это обусловлено рядом причин, основными из которых являются: недостаток знаний о физических свойствах и механизмах процессов тепломассопереноса в тонкопленочных теплоизоляционных покрытиях.
\end{abstract}

Цель: исследование кондуктивного теплопереноса в слое тонкопленочной тепловой изоляции с учетом разнородности свойств микросфер и связующих веществ.

Объекты: цилиндрические слои тонкопленочного теплоизоляционного покрытия. На внутренней и внешней поверхностях теплоизоляционного покрытия поддерживаются постоянные температуры. Рассматривались два варианта геометрии тонкопленочного теплоизоляционного покрытия: “связующее вещество и полнотелые микросферы» и “связующее вещество и полые микросферы». Исследования проводились для слоя теплоизоляции толщиной 0,33мм. Температуры на внутренней и внешней поверхностях изоляции принималась в соответствии с экспериментальными данными. Предполагалось, что слой тонкопленочной теплоизоляции на 62 \% состоит из микросфер диаметром 50 мкм и на 38 \% из связующего вещества. Рассматривались два типа полых микросфер с толщинами стенок: 5 и 2 мкм.

Методы. Экспериментальные исследования проводились с использованием оригинального лабораторного стенда. Решение поставленных задач получено методом конечных элементов. Использовалась аппроксимация Галеркина, неравномерная конечно-элементная сетка. Параметры элементов сетки выбирались из условий сходимости решения. Увеличение числа элементов расчетной сетки проводилось с использованием метода Делоне.

Результаты. Установлено, что средний коэффициент теплопроводности тонкопленочного теплоизоляционного покрытия в диапазоне температур $50-90^{\circ} \mathrm{C}$ составляет 0,0574 Вт/(м•K), что существенно отличается от заявленных фирмой-производителем значений. Выявлено влияние на тепловые потери вида связующего вещества и характеристик микросфер (полые или полнотелые), толщины стенки микросферы и газовой фазы, содержащейся в полости микросферы. Для рассматриваемого случая отклонение от экспериментальных данных составило от 9,36 до 91,12 \% в зависимости от состава тонкопленочного теплоизоляционного покрытия. Такие отклонения обусловлены резким изменением эффективных теплофизических свойств тепловой изоляции при различных характеристиках компонентов тонкопленочной тепловой изоляции. Анализ результатов численного моделирования позволяет сделать вывод о том, что наиболее вероятный состав тонкопленочной теплоизоляции включает в себя полые микросферы и многокомпонентное связующее вещество.

\section{Ключевые слова:}

Тонкопленочная тепловая изоляция, энергосбережение, теплоснабжение,

транспортировка и хранение энергии, моделирование

\section{Введение}

Разработка новых энергосберегающих технологий для тепловой защиты элементов систем теплоснабжения является одной из актуальнейших в настоящее время при создании энергетического оборудования различного назначения [1-4]. Высокий уровень тепловых потерь [5] в системах теплоснабжения (например, тепловые сети, ТЭС, котельные) и энергетическом оборудовании различного назначения (химические производства, пищевая промышленность и т. д.) и неудовлетворительное состояние их тепловой изоляции обосновывают необходимость разработки новых технологий снижения уровня тепловых потерь в рассматриваемых системах.

В настоящее время появляются принципиально новые материалы, в состав которых входят микросферы и различного рода связующие элементы, так называемые тонкопленочные теплоизоляцион- ные покрытия, обладающие свойствами красок с теплоизоляционным эффектом. Значения коэффициентов теплопроводности тонкопленочных теплоизоляционных покрытий варьируются в диапазоне от 0,001 до 0,45 Вт/(м· $K)$ [6]. Уникальные теплофизические характеристики тонкопленочных теплоизоляционных покрытий позволяют использовать их в различных энергетических системах и оборудовании. Несмотря на это, технологии применения тонкопленочных теплоизоляционных покрытий к настоящему моменту времени не получили развития. Это обусловлено рядом причин, основными из которых являются: недостаток знаний о физических свойствах и механизмах процессов тепломассопереноса в тонкопленочных теплоизоляционных покрытиях.

Среди научно-исследовательских работ в области технологий применения тонкопленочных те- 
плоизоляционных покрытий, опубликованных за последние годы, особо следует выделить публикации о завершенной технологии применения прозрачных тонкопленочных теплоизоляционных покрытий [7-11], а также работы об исследовании теплопроводности одиночных [12] и не упорядоченных засыпок [13] полых микросфер.

Также известно большое количество исследований [14-20], в которых обосновывается целесообразность и эффективность применения тонкопленочных теплоизоляционных покрытий в различных энергетических приложениях и развивается новая технология применения быстромонтируемых термостойких многослойных тонкопленочных теплоизоляционных конструкций с учетом влияния некоторых внешних факторов (влажность, паропроницаемость, морозостойкость и другие).

Анализ современной научной литературы, в частности профильных журналов по энергетике и тепломассообмену, показывает, что проблема моделирования тепломассопереноса в тонкопленочных теплоизоляционных покрытиях не решена мировым сообществом до настоящего времени. Известны лишь одиночные исследования $[12,13]$ о моделировании теплопереноса в системах, состоящих из микросфер. Данных об особенностях процессов тепломассообмена, влиянии основных значимых факторов и процессов на интенсификацию тепловых потерь, технологиях применения тонкопленочных теплоизоляционных покрытий в условиях реальной эксплуатации в научной литературе не опубликовано.

Целью работы является исследование кондуктивного теплопереноса в слое тонкопленочной тепловой изоляции с учетом разнородности свойств микросфер и связующих веществ.

\section{Экспериментальное исследование}

Экспериментальное определение тепловых потерь теплопроводов с применением тонкопленочного теплоизоляционного покрытия

Схематическое изображение лабораторного стенда, имитирующего однотрубный теплопровод, использовавшегося при проведении экспериментальных исследований, приведено на рис. 1. Основные элементы лабораторного стенда: трубчатый термоэлектрический нагреватель (ТЭН) - 1 (диаметр 13 мм, длина $1 \mathrm{~m}$ ), слой тонкопленочного теплоизоляционного покрытия - 2. На внешних поверхно- стях ТЭНа и полутолщине слоя изоляции установлены хромелькопелевые термопары - 3. В качестве преобразователя сигнала от термопар использовался измеритель температуры УТК 38Щ4ТП. Термопары использовались для контроля температур поверхности ТЭНа и полутолщины слоя тепловой изоляции, а также индикации установления стационарного режима теплопереноса. Электрические параметры (ток и напряжение) измерялись цифровым мультиметром, а мощность ТЭНа регулировалась лабораторным автотрансформатором. Погрешность измерения температуры составляла $2-3 \%$ и оценивалась по методике [21].

Экспериментальные исследования тепловых потерь теплопровода проводились в следующей последовательности. Первая группа термопар монтировалась на внешнюю поверхность ТЭНа и наносился первый полуслой тепловой изоляции (толщина 0,33 мм). На поверхность первого полуслоя тепловой изоляции симметрично первой группе укладывалась вторая группа термопар. Затем наносился второй слой теплоизоляционного покрытия. В качестве тепловой изоляции использовалось одно из наиболее распространенных в настоящее время тонкопленочных покрытий, бренд которого не раскрывается. Для определения толщины слоя тонкопленочного теплоизоляционного покрытия использовался микрометр типа МК модель 102 (цена деления 0,01, допустимая погрешность $\pm 0,004$, класс точности 2). После подготовки стенда $к$ работе включался электрический нагреватель. Для поддержания на поверхности ТЭНа и полутолщине слоя изоляции постоянных температур по мере установления стационарного режима теплопроводности необходимо было регулировать мощность нагревателя трансформатором.

С целью снижения влияния термопар на искажение температурного поля тонкопленочного теплоизоляционного покрытия термопары первой группы зачеканивались в поверхность ТЭНа, а корольки термопар второй группы выбирались таким образом, чтобы их размеры были минимальными.

Тепловые потери $Q$ определялись с погрешностью $0,5 \%$, обусловленной характеристиками мультиметра, по формуле:

$$
Q=\frac{I U}{L},
$$

где $L$ - длина ТЭНа, м; $I$ - ток, А; $U$ - напряжение, В.

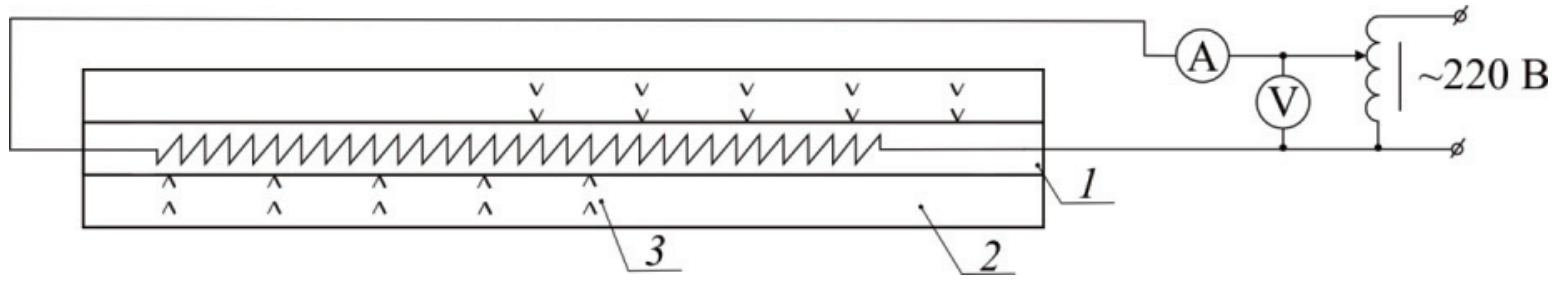

Pис. 1. Схема лабораторного стенда: 1 - ТЭН, 2 - слой тепловой изоляции, 3 - места закладки термопар

Fig. 1. Scheme of laboratory stand: 1 is the tubular thermoelectric heater, 2 is the thermal insulation layer, 3 are the locations of thermocouples 
Определение коэффициента теплопроводности

тонкопленочной тепловой изоляции

Коэффициент теплопроводности тонкопленочного теплоизоляционного покрытия вычислялся по формуле:

$$
\lambda=\frac{Q \ln \left(d_{2} / d_{1}\right)}{2 \pi L\left(T_{4}-T_{3}\right)},
$$

где $d_{2}, d_{1}$ - наружный и внутренний диаметры мест закладки термопар, м; $T_{4}, T_{3}$ - температуры на внутреннем и наружном диаметрах мест закладки термопар.

При фиксированных значениях температур в местах закладки термопар с целью исключения случайной погрешности эксперименты повторялись пять раз в идентичных условиях.

\section{Результаты экспериментальных исследований}

Основные результаты экспериментального исследования тепловых потерь теплопровода, покрытого тонкопленочной тепловой изоляцией, приведены в табл. 1.

Таблица 1. Результаты экспериментального определения тепловых потерь

Table 1. Results of experimental determination of heat loss

\begin{tabular}{|c|c|c|c|}
\hline$T_{3}, \mathrm{~K}$ & 359,49 & 339,59 & 319,77 \\
\hline$T_{4}, \mathrm{~K}$ & 363,15 & 343,15 & 323,15 \\
\hline $\mathrm{Q}, \mathrm{B} \mathrm{T} / \mathrm{M}(\mathrm{W} / \mathrm{m})$ & 27,09 & 25,84 & 24,20 \\
\hline
\end{tabular}

Анализ результатов экспериментального определения тепловых потерь теплопровода, изолированного тонкопленочной тепловой изоляцией, позволяет сделать ожидаемый вывод об увеличении теплового потока с ростом температуры поверхности теплопровода. При этом перепад температур по полутолщине слоя тонкопленочной изоляции составляет около $4^{\circ}$.

На рис. 2 приведены результаты экспериментального определения коэффициента теплопроводности тонкопленочного теплоизоляционного покрытия при различных значениях температуры поверхности ТЭНа.

Результаты исследования, приведенные на рис. 2 , позволяют сделать вывод о том, что теплопроводность тонкопленочного теплоизоляционного покрытия возрастает с увеличением температуры поверхности ТЭНа. Доверительный интервал для всех вариантов экспериментов составляет $\pm 0,0002 \mathrm{BT} /(\mathrm{m} \cdot K)$. Теплопроводность тонкопленочного теплоизоляционного покрытия в рассматриваемом диапазоне температур изменяется от 0,0565 до 0,0584 $\mathrm{BT} /(\mathrm{m} \cdot \mathrm{K})$, что составляет $3,25 \%$. По этой причине в практических расчетах теплопроводность тонкопленочных теплоизоляционных материалов можно принимать постоянной.

Средний коэффициент теплопроводности тонкопленочного теплоизоляционного покрытия в диапазоне температур $50-90{ }^{\circ} \mathrm{C}$ составил $0,0574 \mathrm{BT} /(\mathrm{m} \cdot \mathrm{K})$, что существенно отличается от заявленных фирмой-производителем значений [6].

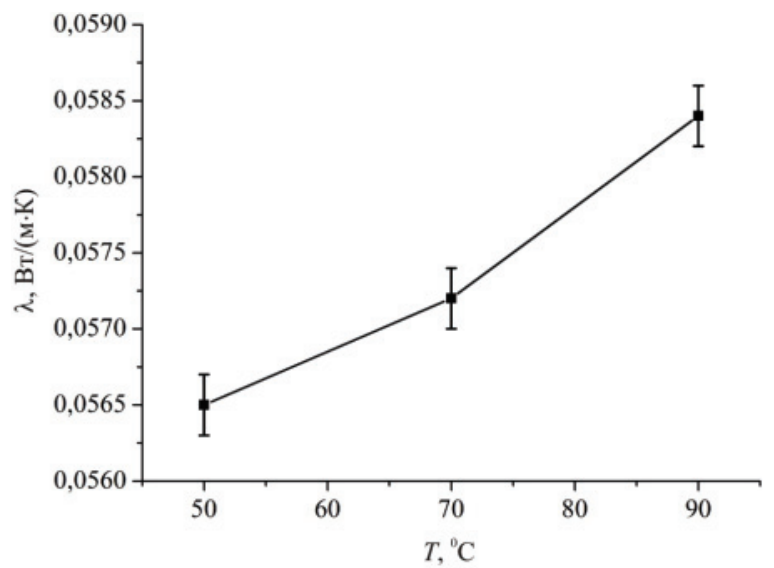

Рис. 2. Зависимость теплопроводности тонкопленочного теплоизоляиионного покрытия от телпературы

Fig. 2. Dependence of thermal conductivity of thin-film heat-insula ting coating on temperature

Из [14-16] известно, что теплопроводность тонкопленочной теплоизоляции составляет 0,028 Вт/(м•К) при температуре $60{ }^{\circ} \mathrm{C}$ и 0,036 Вт $/(\mathrm{s} \cdot К)$ при температуре $100{ }^{\circ} \mathrm{C}$ для покрытий с вакуумированными микросферами и 0,14 и $0,19 \mathrm{BT} /(\mathrm{M} \cdot \mathrm{K})$ для газонаполненных микросфер. Поскольку фактический состав исследуемого материала не известен, провести детализированное сопоставление результатов исследований не представляется возможным. Полученное в настоящей работе значение коэффициента теплопроводности находится в указанном выше диапазоне, что косвенно подтверждает адекватность полученных результатов.

Численное моделирование теплопереноса

\section{в тонкопленочных теплоизоляционных покрытиях}

Постановка задачи

На рис. 3 приведены схематичные изображения областей решения задач. Рассматривается слой тонкопленочного теплоизоляционного покрытия. Предполагается, что на внутренней и внешней поверхностях изоляционного слоя поддерживаются постоянные температуры.

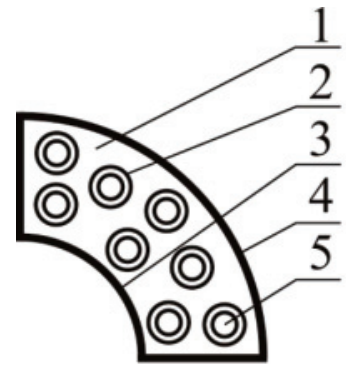

$a$

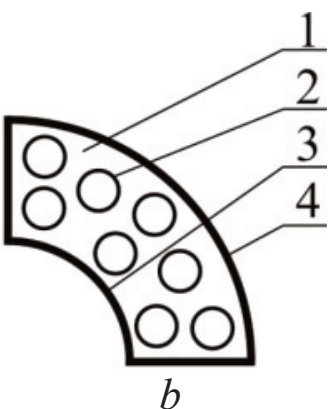

$b$
Pис. 3. Схематичное изображение области решения: 1 - связую щее вещество; 2 - микросферы; 3 - внутренняя поверх ность изоляии; 4 - внешнял поверхность изоляиин; 5 полость микросферы

Fig. 3. Scheme of solution area: 1 is the binder; 2 are the microspheres; 3 is the inner surface of insulation; 4 is the outer surface of insulation; 5 is the microsphere cavity 
Рассматривались два варианта геометрии тонкопленочного теплоизоляционного покрытия:

1. Связующее вещество и полнотелые микросферы (рис. 3,a).

2. Связующее вещество и полые микросферы (рис. 3, b).

Основные допущения, принятые при постановке задачи, заключаются в следующем:

1. Теплофизические свойства материалов и веществ имеют известные и постоянные значения. Диапазон изменения параметров, влияющих на теплофизические характеристики, в рассматриваемой задаче невелик [22], следовательно, изменением свойств можно пренебречь.

2. На границах контакта между микросферами и связующим веществом выполняются условия идеального теплового контакта.

3. Не учитывается теплообмен излучением и фазовые переходы на внешней поверхности тонкопленочного теплоизоляционного покрытия.

4. Теплоперенос в рассматриваемых областях решения (рис. 3) реализуется только за счет теплопроводности.

5. Микросферы имеют одинаковые геометрические характеристики, а их размещение в изоляционном слое является упорядоченным.

\section{Математическая модель}

Процесс переноса тепла для рассматриваемого случая будет описываться следующими выражениями:

$$
\begin{gathered}
\nabla^{2} T_{1}=0 ; \\
\nabla^{2} T_{2, i}=0 ; i=1-n, \\
\nabla^{2} T_{5, i}=0 ; i=1-n . \\
T_{3}=\text { const, } \\
T_{4}=\text { const. } \\
\lambda_{1} \operatorname{grad}\left(T_{1}\right)=\lambda_{2} \operatorname{grad}\left(T_{2, i}\right) ; T_{1}=T_{2, i}, \\
\lambda_{2} \operatorname{grad}\left(T_{2, i}\right)=\lambda_{5} \operatorname{grad}\left(T_{5, i}\right) ; T_{2, i}=T_{5, i} .
\end{gathered}
$$

Обозначения: $\lambda$ - теплопроводность, Вт/(м.К); $T$ - температура, $\mathrm{K} ; n$ - количество микросфер, шт; индексы 1-5 - в соответствии с рис. 3.

\section{Метод решения и исходные данные}

При решении задачи (1)-(7) использовался метод конечных элементов с аппроксимацией Галеркина [23]. Моделирование проводилось на неравномерной конечно-элементной сетке. Количество элементов выбиралось из условий сходимости решения; сгущение сетки проводилось методом Делоне [23].

Численное моделирование проводилось для слоя теплоизоляции толщиной 0,33 мм. Температура на внутренней и внешней поверхностях изоляции принималась в соответствии с экспериментальными данными (табл. 1). Предполагалось, что слой тонкопленочной теплоизоляции на $62 \%$ состоит из микросфер диаметром 50 мкм и на 38 \% из связую- щего вещества. Рассматривались два типа полых микросфер с толщинами стенок: 5 и 2 мкм. Микросферы по толщине тонкопленочного теплоизоляционного покрытия располагались упорядоченно с «коридорным» вариантом размещения.

Фактический состав тонкопленочных теплоизоляционных материалов является коммерческой тайной и не раскрывается производителями. По этой причине дополнительной задачей в настоящем исследовании являлся параметрический поиск характеристик микросфер и связующих веществ.

В табл. 2 приведены значения $[22,24]$ теплопроводности $(\lambda)$, плотности $(\rho)$ и теплоемкости $(c)$

\begin{tabular}{|c|c|c|c|c|}
\hline \multicolumn{2}{|c|}{$\begin{array}{c}\text { Характеристика } \\
\text { Characteristic }\end{array}$} & $\begin{array}{l}\lambda, \mathrm{BT} /(\mathrm{M} \cdot \mathrm{K}) \\
(\mathrm{W} /(\mathrm{m} \cdot \mathrm{K}))\end{array}$ & $\begin{array}{l}\rho, \mathrm{Кг} / \mathrm{M}^{3} \\
\left(\mathrm{~kg} / \mathrm{m}^{3}\right)\end{array}$ & $\begin{array}{c}c, \text { Дж/кг } \cdot \mathrm{K} \\
(\mathrm{J} /(\mathrm{kg} \cdot \mathrm{K}))\end{array}$ \\
\hline \multirow{3}{*}{$\begin{array}{c}\text { Материал } \\
\text { микросфер } \\
\text { Microsphere } \\
\text { material }\end{array}$} & $\begin{array}{c}\text { Стекло C38-1 } \\
\text { Glass C38-1 }\end{array}$ & 0,92 & 3000 & 650 \\
\hline & $\begin{array}{l}\text { Стекло C39-1 } \\
\text { Glass C39-1 }\end{array}$ & 1,30 & 2800 & 650 \\
\hline & $\begin{array}{c}\text { Стекло C41-1 } \\
\text { Glass C41-1 }\end{array}$ & 0,84 & 3000 & 650 \\
\hline \multirow{2}{*}{$\begin{array}{c}\text { Связующее } \\
\text { вещество } \\
\text { Binder }\end{array}$} & $\begin{array}{c}\text { Однокомпонентное } \\
\text { Single component }\end{array}$ & 0,175 & 1030 & 840 \\
\hline & \begin{tabular}{|l|} 
Tрехкомпонентное \\
Three-component
\end{tabular} & 0,048 & 1060 & 840 \\
\hline \multirow{2}{*}{$\begin{array}{c}\text { Газовая фаза } \\
\text { Gas phase }\end{array}$} & Воздух/Air & 0,029 & 1,0985 & 1007 \\
\hline & $\mathrm{CO}_{2}+\mathrm{N}_{2}$ & 0,031 & 1,1 & 1040 \\
\hline
\end{tabular}
компонентов тонкопленочного теплоизоляционного покрытия, использовавшиеся при проведении исследований тепловых режимов рассматриваемых систем (рис. 3).

Таблица 2. Теплофизические характеристики [22, 24]

Table 2. Thermophysical characteristics [22, 24]

\section{Результаты численного анализа}

В табл. 3-5 приведены величины тепловых потерь теплопровода, покрытого слоем тонкопленочной тепловой изоляции, в зависимости от состава материала (табл. 2), вычисленные в соответствии исходными экспериментальными данными (табл. 1), а также приведено сопоставление результатов численного моделирования и эксперимента. Через знак «/» указаны значения для трех- и однокомпонентного связующего вещества соответственно. Тепловые потери для системы «полнотелые микросферы и связующее вещество» указаны только для однокомпонентного связующего.

Результаты численного моделирования, приведенные в табл. 3-5, позволяют сделать вывод о существенном влиянии состава тонкопленочного теплоизоляционного покрытия на тепловые потери теплопровода.

Сопоставление результатов численного моделирования тепловых потерь теплопровода, изолированного тонкопленочным теплоизоляционным материалом, с результатами экспериментальных исследований свидетельствует об их существенном расхождении (от 9,36 до $91,12 \%$ ) в зависимости от состава тонкопленочного теплоизоляционного покрытия. 
Таблииа 3. Результаты численного моделирования теплопереноса в слое тонкопленочной тепловой изоляции при $T_{4}=359,49 \mathrm{~K}, \mathrm{~T}_{3}=363,15 \mathrm{~K}$

Table 3. Results of numerical simulation of heat transfer in the layer of thin-film thermal insulation at $T_{4}=359,49 \mathrm{~K}$, $T_{3}=363,15 \mathrm{~K}$

\begin{tabular}{|c|c|c|c|}
\hline \multicolumn{2}{|c|}{$\begin{array}{c}\text { Состав тонкопленочного } \\
\text { теплоизоляционного покрытия } \\
\text { Composition of the thin-film } \\
\text { insulation coating }\end{array}$} & $\begin{array}{c}Q, \mathrm{BT} / \mathrm{M} \\
(\mathrm{W} / \mathrm{m})\end{array}$ & \begin{tabular}{|} 
Расхождение с \\
эксперименталь- \\
ными данными, \% \\
Discrepancy with \\
experimental da-
\end{tabular} \\
\hline \multirow{3}{*}{$\begin{array}{l}\text { Полнотелые } \\
\text { микросферы } \\
\text { Full-bodied } \\
\text { microspheres }\end{array}$} & \begin{tabular}{|c|} 
Стекло C38-1 \\
Glass C38-1
\end{tabular} & 178,56 & 88,52 \\
\hline & \begin{tabular}{|c|} 
Стекло C39-1 \\
Glass C39-1
\end{tabular} & 206,24 & 87,47 \\
\hline & $\begin{array}{c}\text { Стекло C41-1 } \\
\text { Glass C41-1 }\end{array}$ & $86,24 / 171,36$ & $68,55 / 84,92$ \\
\hline \multirow{2}{*}{$\begin{array}{c}\text { Полые микросферы } \\
\text { с толщиной стенки } \\
5 \text { мкм } \\
\text { Hollow microspheres } \\
\text { with a wall thickness } \\
\text { of } 5 \text { microns }\end{array}$} & Воздух/Air & $52,08 / 75,36$ & $47,91 / 65,7$ \\
\hline & $\mathrm{CO}_{2}+\mathrm{N}_{2}$ & $54,12 / 74,92$ & $49,83 / 65,51$ \\
\hline \multirow{2}{*}{$\begin{array}{c}\text { Полые микросферы } \\
\text { с толщиной стенки } \\
2 \text { мкм } \\
\text { Hollow microspheres } \\
\text { with a wall thickness } \\
\text { of } 2 \text { microns }\end{array}$} & Воздух/Air & $29,89 / 47,33$ & $9,36 / 45,4$ \\
\hline & $\mathrm{CO}_{2}+\mathrm{N}_{2}$ & $33,08 / 46,60$ & $17,9 / 44,31$ \\
\hline
\end{tabular}

На рис. 4,5 , в качестве примеров, представлены фрагменты типичных температурных полей в рассматриваемых областях решения.

Распределения температур, приведенные на рис. 4,5 , демонстрируют деформацию температурного поля, вызванную разнородностью теплофизи- ческих характеристик тонкопленочных теплоизоляционных покрытий в зависимости от композиционного состава материала. На рис. 4,5 отчетливо видны изменения в направлениях изотермических линий, что соответствует представлениям 0 процессах теплопроводности в твердых телах.

таблица 4. Результаты численного моделирования теплопереноса в слое тонкопленочной тепловой изоляиии при $T_{4}=339,59 \mathrm{~K}, T_{3}=343,15 \mathrm{~K}$

Table 4. Results of numerical simulation of heat transfer in the layer of thin-film thermal insulation at $T_{4}=339,59 \mathrm{~K}$, $T_{3}=343,15 \mathrm{~K}$

\begin{tabular}{|c|c|c|c|}
\hline \multicolumn{2}{|c|}{$\begin{array}{c}\text { Состав тонкопленочного } \\
\text { теплоизоляционного покрытия } \\
\text { Composition of the thin-film } \\
\text { insulation coating }\end{array}$} & $\begin{array}{c}Q, \mathrm{~B} \mathrm{~T} / \mathrm{M} \\
(\mathrm{W} / \mathrm{m})\end{array}$ & $\begin{array}{c}\text { Расхождение } \\
\text { с эксперименталь- } \\
\text { ными данными, \% } \\
\text { Discrepancy with ex- } \\
\text { perimental data, \% }\end{array}$ \\
\hline \multirow{3}{*}{$\begin{array}{l}\text { Полнотелые } \\
\text { микросферы } \\
\text { Full-bodied } \\
\text { microspheres }\end{array}$} & \begin{tabular}{|c|} 
Стекло C38-1 \\
Glass C38-1
\end{tabular} & 198,72 & 86,37 \\
\hline & \begin{tabular}{|c|} 
Стекло C39-1 \\
Glass C39-1
\end{tabular} & 229,44 & 88,2 \\
\hline & \begin{tabular}{|c|} 
Стекло C41-1 \\
Glass C41-1
\end{tabular} & $83,88 / 190,64$ & $68,86 / 85,79$ \\
\hline \multirow{2}{*}{$\begin{array}{c}\text { Полые микросферы } \\
\text { с толщиной стенки } \\
5 \text { мкм } \\
\text { Hollow microsphe- } \\
\text { res with a wall thic- } \\
\text { kness of } 5 \text { microns }\end{array}$} & $\begin{array}{c}\text { Воздух } \\
\text { Air }\end{array}$ & $50,66 / 83,84$ & $48,32 / 67,69$ \\
\hline & $\mathrm{CO}_{2}+\mathrm{N}_{2}$ & $53,78 / 83,2$ & $48,14 / 67,44$ \\
\hline \multirow{2}{*}{\begin{tabular}{|c|} 
Полые микросферы \\
с толщиной стенки \\
2 мкм \\
Hollow microsphe- \\
res with a wall thic- \\
kness of 2 microns
\end{tabular}} & Воздух Air & $28,78 / 52,64$ & $10,2 / 48,54$ \\
\hline & $\mathrm{CO}_{2}+\mathrm{N}_{2}$ & $33,08 / 46,60$ & $17,9 / 44,31$ \\
\hline
\end{tabular}

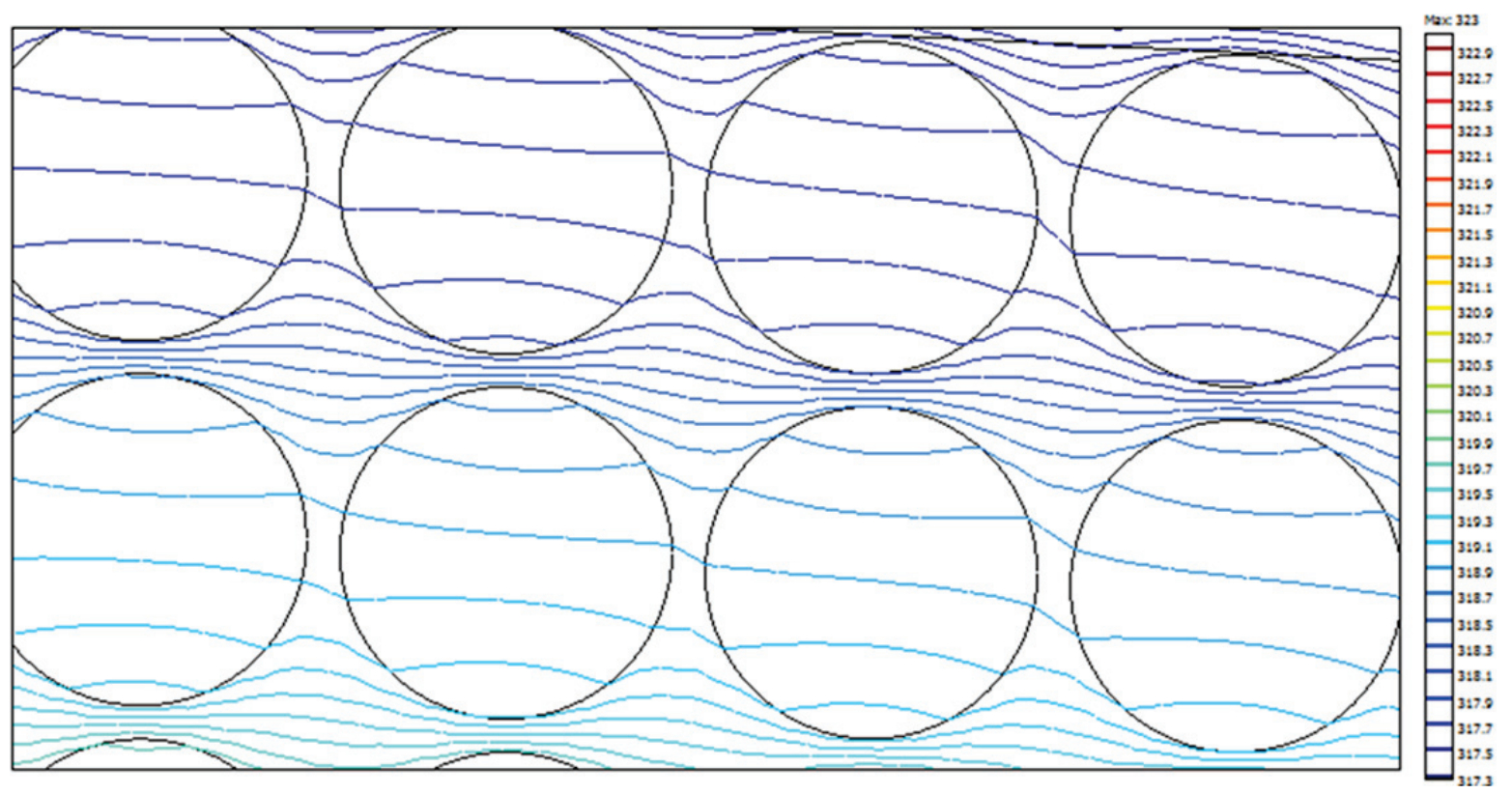

Рис.4. Фраглент распределения температур в слое тонкопленочной теплоизоляции (полнотелье микросферы и одноколпонентное связующее вещество)

Fig. 4. Fragment of temperature distribution in the thin-film thermal insulation layer (solid microspheres and one-component binder) 


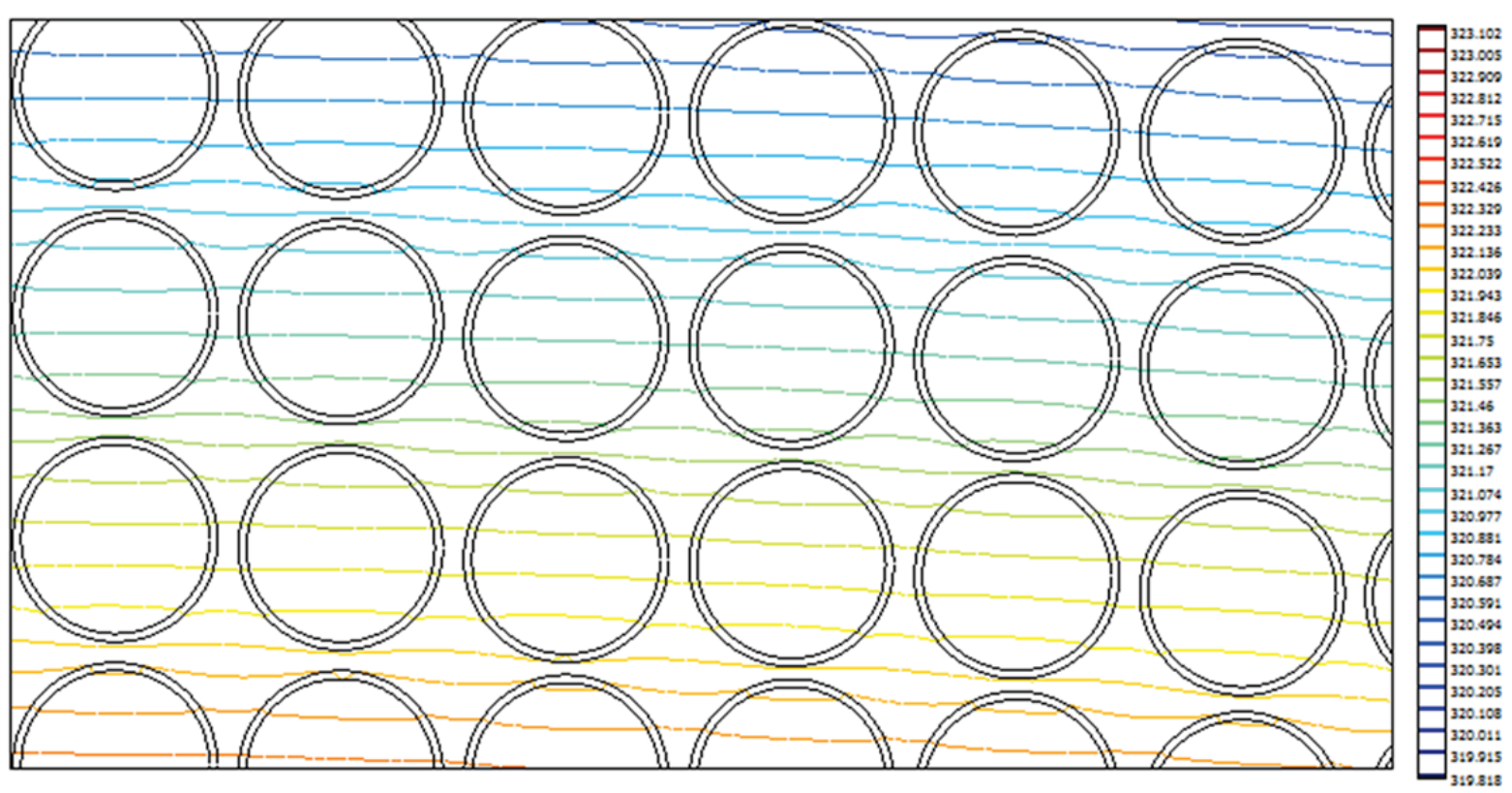

Рис. 5. Фраглент распределения температур в слое тонкопленочной теплоизоляции (полье микросферы с толщиной стенки 5 лкли и однокомпонентное связующее вещество)

Fig. 5. Fragment of temperature distribution in the layer of thin-film thermal insulation (hollow microspheres with a wall thickness of 5 micrometers and a single-component binder)

Таблица 5. Результаты численного моделирования теплопере носа в слое тонкопленочной тепловой изоляции при $\mathrm{T}_{4}=319,77 \mathrm{~K}, \mathrm{~T}_{3}=323,15 \mathrm{~K}$

Table 5. Results of numerical simulation of heat transfer in the layer of thin-film thermal insulation at $T_{4}=319,77 \mathrm{~K}$, $T_{3}=323,15 \mathrm{~K}$

\begin{tabular}{|c|c|c|c|}
\hline \multicolumn{2}{|c|}{$\begin{array}{c}\text { Состав тонкопленочного } \\
\text { теплоизоляционного покрытия } \\
\text { Composition of the thin-film } \\
\text { insulation coating }\end{array}$} & $\begin{array}{c}Q, \mathrm{~B} \text { T/M } \\
(\mathrm{W} / \mathrm{m})\end{array}$ & $\begin{array}{l}\text { Расхождение } \\
\text { с эксперименталь- } \\
\text { ными данными, \% } \\
\text { Discrepancy with ex- } \\
\text { perimental data, \% }\end{array}$ \\
\hline \multirow{3}{*}{$\begin{array}{c}\text { Полнотелые } \\
\text { микроссферы } \\
\text { Full-bodied } \\
\text { microspheres }\end{array}$} & \begin{tabular}{|c|} 
Стекло С $38-1$ \\
Glass C38-1
\end{tabular} & 316 & 92,34 \\
\hline & \begin{tabular}{|c|} 
Стекло C39-1 \\
Glass C39-1
\end{tabular} & 364,8 & 93,37 \\
\hline & $\begin{array}{c}\text { Стекло C41-1 } \\
\text { Glass C41-1 }\end{array}$ & $79,63 / 272,8$ & $69,36 / 91,12$ \\
\hline \multirow{2}{*}{$\begin{array}{c}\text { Полые микросферы } \\
\text { с толщиной стенки } \\
5 \text { мкм } \\
\text { Hollow microspheres } \\
\text { with a wall thickness } \\
\text { of } 5 \text { microns }\end{array}$} & $\begin{array}{c}\text { Воздух } \\
\text { Air }\end{array}$ & $48,03 / 120$ & $49,59 / 79,84$ \\
\hline & $\mathrm{CO}_{2}+\mathrm{N}_{2}$ & $51,99 / 119,2$ & $49,66 / 79,7$ \\
\hline \multirow{2}{*}{$\begin{array}{c}\text { Полые микросферы } \\
\text { с толщиной стенки } \\
2 \text { мкм } \\
\text { Hollow microspheres } \\
\text { with a wall thickness } \\
\text { of } 2 \text { microns }\end{array}$} & Воздух Air & $28,12 / 75,2$ & $13,57 / 67,8$ \\
\hline & $\mathrm{CO}_{2}+\mathrm{N}_{2}$ & $30,56 / 74,08$ & $20,81 / 67,34$ \\
\hline
\end{tabular}

\section{Заключение}

Проведено исследование теплопереноса в слое тонкопленочной тепловой изоляции с учетом разнородности свойств микросфер и связующих веществ.
Установлено, что средний коэффициент теплопроводности тонкопленочного теплоизоляционного покрытия в диапазоне температур $50-90{ }^{\circ} \mathrm{C}$ составляет $0,0574 \mathrm{Bт} /($ м $К$ К), что существенно отличается от заявленных фирмой-производителем значений.

Выявлено влияние на тепловые потери вида связующего вещества и характеристик микросфер (полые или полнотелые), толщины стенки микросферы и газовой фазы, содержащейся в полости микросферы. Для рассматриваемого случая отклонение от экспериментальных данных составило от 9,36 до $91,12 \%$ в зависимости от состава тонкопленочного теплоизоляционного покрытия. Такие отклонения обусловлены резким изменением эффективных теплофизических свойств тепловой изоляции при различных характеристиках компонентов тонкопленочной тепловой изоляции.

Показано, что применение полых микросфер для тонкопленочной теплоизоляции по сравнению с полнотелыми микросферами приводит к более существенному снижению тепловых потоков. Это обстоятельство объясняется, прежде всего, значениями теплофизических характеристик связующего вещества и газа-наполнителя полых микросфер.

Анализ результатов численного моделирования позволяет сделать вывод о том, что наиболее вероятный состав тонкопленочной теплоизоляции включает в себя полые микросферы и многокомпонентное связующее вещество.

Исследование выполнено при финансовой поддержке РФФИ в рамках научного проекта № 18-48-700008-p_a. 


\section{СПИСОК ЛИТЕРАТУРЫ}

1. Optimization modeling for smart operation of multi-source district heating with distributed variable-speed pumps / H. Wang, H. Wang, Zh. Haijian, T. Zhu // Energy. - 2017. - V. 138. P. $1247-1262$.

2. Duquette J., Rowe A., Wild P. Thermal performance of a steady state physical pipe model for simulating district heating grids with variable flow // Applied Energy. - 2016. - V. 178. P. 383-393.

3. Thermal transient prediction of district heating pipeline: Optimal selection of the time and spatial steps for fast and accurate calculation / Y. Wanga, S. Youa, H. Zhanga, X. Zhenga, W. Zhenga, Q. Miaoa, G. Luc // Applied Energy. - 2017. - V. 206. P. $900-910$.

4. Ertürk M. Optimum insulation thicknesses of pipes with respect to different insulation materials, fuels and climate zones in Turkey // Energy. - 2016. - V. 113. - P. 991-1003.

5. Половников В.Ю. Тепловые режимы и тепловые потери подземных трубопроводов с учетом реальных условий теплообмена на внешнем контуре взаимодействия // Известия Томского политехнического университета. Инжиниринг георесурсов. 2018. - T. 329. - № 1. -C. 124-131.

6. Об эффективности применения тонкопленочных теплоизоляционных покрытий / В.А. Рыженков, Н.А. Логинова, А.И. Бычков, А.Ф. Прищепов // Научное обозрение. - 2013. № 5. - C. 71-75.

7. Chou H.M., Chen C.R., Wu T.N. New transparent thin films for thermal insulation // Journal of the Chinese Society of Mechanical Engineers. Transactions of the Chinese Institute of Engineers. Series C. - 2015. - V. 36 (1). - P. 85-90.

8. The evaluation of energy saving using ultrathin heat insulation in railway electrification substation / H. Kim, J. Jang, S. Shin, Y. Park, S. Kim, H.R. Kim, B. Hyun, J. Kim // Transactions of the Korean Institute of Electrical Engineers. - 2015. V. 64 (1). - P. 171-175.

9. Qi Y., Yin X., Zhang J. Transparent and heat-insulation plasticized polyvinyl chloride (PVC) thin film with solar spectrally selective property // Solar Energy Materials and Solar Cells. - 2016. V. 151 . - P. 30-35.

10. Patankar S.N., Kranov Y.A. Hollow glass microsphere HDPE composites for low energy sustainability // Materials Science and Engineering. - 2010. - V. 527. - P. 1361-1366.

11. Effect of hollow glass microsphere thin film on heat-insulation performance of building envelope / H. Wang, H.J. Fang, Q.D. Lan, S. He, C. Jiang // Shanghai Jiaotong Daxue Xuebao/Journal of Shanghai Jiaotong University. - 2014. V. 48 (9). - P. 1341-1345.
12. Thermal conductivity of powder silica hollow spheres / Y. Liao, X. Wua, H. Liua, Y. Chen // Thermochimica Acta. - 2011. V. 526. - P. 178-184.

13. Numerical analyses of the thermal conductivity of random hollow sphere structures / T. Fiedler, R. Löffler, T. Bernthaler, R. Winkler, I.V. Belova, G.E. Murch, A. Öchsnerc // Materials Letters. - 2009. - V. 63. - № 13-14. - P. 1125-1127.

14. 0 возможности использования микросфер с модифицированной поверхностью в составе тонкопленочных теплоизоляционных покрытий / В.А. Рыженков, Н.А. Логинова, А.И. Бычков, А.Ф. Прищепов // Энергосбережение и водоподготовка. 2013. - № 4. - C. 21-23.

15. Рыженков В.А., Логинова Н.А., Прищепов А.Ф. Эффективность тонкопленочных теплоизоляционных покрытий при использовании микросфер для систем теплоснабжения // Надежность и безопасность энергетики. - 2012. - № 17. - С. 61-63.

16. Испытания тонкопленочных многослойных теплоизоляционных покрытий на морозостойкость / Н.А. Логинова, А.В. Рыженков, А.Ф. Прищепов, Е.Е. Лапин, А.Ю. Плещева // Надежность и безопасность энергетики. - 2014. - № 2. C. $23-25$.

17. Исследование влияния влаги на теплоизоляционные свойства полых микросфер / В.В. Беккер, С.В. Григорьев, Н.А. Логинова, С.И. Погорелов, А.В. Рыженков, А.В. Аникеев // Нефтяное хозяйство. - 2015. - № 12. - С. 136-137.

18. 0 результатах исследований паропроницаемости новых видов теплоизоляционных материалов / Е.В. Беляева, Н.А. Логинова, Е.Е. Лапин, С.И. Погорелов, А.В. Рыженков // Нефтяное хозяйство. - 2015. - № 8. - С. 114-116.

19. Выбор и оптимизация соотношения компонентов для разработки быстромонтируемых термостойких теплоизоляционных конструкций / Н.А. Логинова, С.В. Григорьев, Е.Е. Лапин, С.И. Погорелов, А.В. Рыженков // Теплоэнергетика. - 2016. № 5. - C. 51-55.

20. Изучение влияния модификации стеклянных микросфер на свойства синтактных пен на основе олигометилсилоксана / В.С. Осипчик, Н.В. Костромина, Ю.В. Олихова, В.Н. Ивашкина, Е.В. Беляева, Н.А. Логинова, А.В. Рыженков, Е.Е. Лапин // Пластические массы. - 2015. - № 5-6. - С. 36-39.

21. Новицкий П.В., Зограф И.А. Оценка погрешностей результатов измерений. - Л.: Энергоиздат, 1991. - 304 с.

22. Гува А.Я. Краткий теплофизический справочник. - Новосибирск: Сибвузиздат, 2002. - 300 c.

23. Garcia A.L. Numerical methods for physics. - San Jose CA: CreateSpace Independent Publishing Platform, 2015. - $432 \mathrm{c}$.

24. Бабичев Н.А. Физические величины: справочник. - М.: Энергоатомиздат, 1991. - $1232 \mathrm{c.}$

Поступила 28.11.2018 г.

\section{Информация об авторах}

Половников В.Ю., кандидат технических наук, доцент Инженерной школы энергетики Национального исследовательского Томского политехнического университета. 


\title{
CONDUCTIVE HEAT TRANSFER IN LAYER OF THIN-FILM THERMAL INSULATION
}

\author{
Viacheslav Yu. Polovnikov, \\ polov@tpu.ru \\ National Research Tomsk Polytechnic University, \\ 30, Lenin Avenue, Tomsk, 634050, Russia.
}

The relevance of the study is caused by the need to create new energy-saving technologies for thermal protection of elements of heat
supply systems and other energy equipment for various purposes. The high level of heat losses in heat supply systems (for example,
heat networks, thermal power plants, boiler) and power equipment for various purposes (chemical production, food industry, etc.) and
the unsatisfactory condition of their thermal insulation justify the need to develop new technologies to reduce heat losses in the systems
in question. The unique thermophysical characteristics of thin-film thermal insulation coatings allow their use in various energy systems
and equipment. Despite this, the technology of using thin-film heat-insulating coatings has not been developed. This is due to several
reasons, the main of which are: lack of knowledge about the physical properties and mechanisms of heat and mass transfer processes
in thin-film heat-insulating coatings. The main aim of the research is a study of conductive heat transfer in the layer of thin-film thermal insulation taking into account the heterogeneity of the properties of microspheres and binders.

Objects of the research are cylindrical layers of thin-film thermal insulation. It is assumed that constant temperatures are maintained on the inner and outer surfaces. Two variants of the geometry of the thin-film heat-insulating coating were considered: «binder and fullbodied microspheres» and "binder and hollow microspheres». The studies were conducted for 0,33 mm insulation layer. The temperatures on the inner and outer surfaces of the insulation were taken in accordance with the experimental data. It was assumed that $62 \%$ of the thin-film thermal insulation layer consists of microspheres with a diameter of 50 micrometers and $38 \%$ of a binder. Two types of hollow microspheres with wall thicknesses were considered: 5 and 2 micrometers.

Methods. The solution of the problems posed is obtained by the finite element method. Galerkin approximation, non-uniform finite element mesh were used. The parameters of the grid elements were chosen from the conditions of convergence of the solution. An increase in the number of elements of the computational grid was carried out using the Delaunay method. Experimental studies were carried out with the original laboratory stand.

Results. It was established that the average thermal conductivity coefficient of a thin-film heat-insulating coating in the temperature range of $50-90{ }^{\circ} \mathrm{C}$ is $0,0574 \mathrm{~W} /(\mathrm{m} \cdot \mathrm{K})$, which differs significantly from the value stated by the manufacturer. The effect on the thermal losses of the type of binder and the characteristics of the microspheres (hollow or full-bodied), the wall thickness of the microsphere and the gas phase contained in the cavity of the microsphere is revealed. For the case under consideration, the deviation from the experimental data ranged from 9,36 to 91,12\%, depending on the composition of the thin-film thermal insulation coating. This is due to a sharp change in the effective thermophysical properties of thermal insulation with different characteristics of the components of thinfilm thermal insulation. Analysis of the results of numerical simulation allows us to conclude that the most likely composition of thin-film thermal insulation includes hollow microspheres and a multicomponent binder.

\section{Key words:}

Thin-film thermal insulation, energy saving, heat supply, transportation and storage of energy, modeling.

The research was financially supported by the RFBR within the science project no. 18-48-700008-p_a.

\section{REFERENCES}

1. Wang H., Wang H., Haijian Zh., Zhu T. Optimization modeling for smart operation of multi-source district heating with distributed variable-speed pumps. Energy, 2017, vol. 138, pp. 1247-1262.

2. Duquette J., Rowe A., Wild P. Thermal performance of a steady state physical pipe model for simulating district heating grids with variable flow. Applied Energy, 2016, vol. 178, pp. 383-393.

3. Wanga Y., Youa S., Zhanga H., Zhenga X., Zhenga W., Miaoa Q., Luc G. Thermal transient prediction of district heating pipeline: Optimal selection of the time and spatial steps for fast and accurate calculation. Applied Energy, 2017, vol. 206, pp. 900-910.

4. Ertürk M. Optimum insulation thicknesses of pipes with respect to different insulation materials, fuels and climate zones in Turkey. Energy, 2016, vol. 113, pp. 991-1003.

5. Polovnikov V.Y. Thermal regimes and thermal losses of underground pipelines in real heat exchange on the outer interaction boundary. Bulletin of the Tomsk Polytechnic University. Geo Assets Engineering, 2018, vol. 329, no. 1, pp. 124-131. In Rus.

6. Ryzhenkov V.A., Loginova N.A., Bychkov A.I., Prishchepov A.F. On the effectiveness of thin-film insulation coatings. Scientific Review, 2013, no. 5, pp. 71-75. In Rus.
7. Chou H.M., Chen C.R., Wu T.N. New transparent thin films for thermal insulation. Journal of the Chinese Society of Mechanical Engineers. Transactions of the Chinese Institute of Engineers. Series $C$, 2015, vol. 36 (1), pp. 85-90.

8. Kim H., Jang J., Shin S., Park Y., Kim S., Kim H.R., Hyun B., Kim J. The evaluation of energy saving using ultrathin heat insulation in railway electrification substation. Transactions of the Korean Institute of Electrical Engineers, 2015, vol. 64 (1), pp. 171-175.

9. Qi Y., Yin X., Zhang J. Transparent and heat-insulation plasticized polyvinyl chloride (PVC) thin film with solar spectrally selective property. Solar Energy Materials and Solar Cells, 2016, vol. 151 , pp. $30-35$.

10. Patankar S.N., Kranov Y.A. Hollow glass microsphere HDPE composites for low energy sustainability. Materials Science and Engineering, 2010, vol. 527, pp. 1361-1366.

11. Wang H., Fang H.J., Lan Q.D., He S., Jiang C. Effect of hollow glass microsphere thin film on heat-insulation performance of building envelope. Shanghai Jiaotong Daxue Xuebao/Journal of Shanghai Jiaotong University, 2014, vol. 48 (9), pp. 1341-1345.

12. Liao Y., Wua X., Liua H., Chen Y. Thermal conductivity of powder silica hollow spheres. Thermochimica Acta, 2011, vol. 526, pp. 178-184. 
13. Fiedler T., Löffler R., Bernthaler T., Winkler R., Belova I.V., Murch G.E., Öchsnerc A. Numerical analyses of the thermal conductivity of random hollow sphere structures. Materials Letters, 2009, vol. 63, no. 13-14, pp. 1125-1127.

14. Ryzhenkov V.A., Loginova N.A., Bychkov A.I., Prishchepov A.F. On the possibility of using microspheres with a modified surface as part of thin-film heat-insulating coatings. Energy Saving and Water Treatment, 2013, no. 4, pp. 21-23. In Rus.

15. Ryzhenkov V.A., Loginova N.A., Prishchepov A.F. The effectiveness of thin-film insulating coatings when using microspheres for heat supply systems. Reliability and safety of energy, 2012, no. 17, pp. 61-63. In Rus.

16. Loginova N.A., Ryzhenkov A.V., Prishchepov A.F., Lapin E.E., Pleshcheva A.Yu. Tests of thin-film multilayer heat-insulating coatings for frost resistance. Reliability and safety of energy, 2014, no. 2, pp. 23-25. In Rus.

17. Bekker V.V., Grigorev S.V., Loginova N.A., Pogorelov S.I., Ryzhenkov A.V., Anikeev A.V. Study of the effect of moisture on the insulating properties of hollow microspheres. Oil industry, 2015, no. 12, pp. 136-137. In Rus.

18. Beliaeva E.V., Loginova N.A., Lapin E.E., Pogorelov S.I., Ryzhenkov A.V. On the results of vapor permeability studies of new types of thermal insulation materials. Oil industry, 2015, no. 8, pp. 114-116. In Rus.
19. Loginova N.A., Grigorev S.V., Lapin E.E., Pogorelov S.I, Ryzhenkov A.V. Choice and optimization of ratio of components to develop fast-mounted thermostable heat-insulating constructions. Thermal Engineering, 2016, vol. 63, no. 5, pp. 355-359. In Rus.

20. Osipchik V.S., Kostromina N.V., Olihova Iu.V., Ivashkina V.N., Beliaeva E.V., Loginova N.A., Ryzhenkov A.V., Lapin E.E. Studying the effect of modifying glass microspheres on the properties of oligomethylsiloxane-based syntactic foams. Plastics, 2015, no. 5-6, pp. 36-39. In Rus.

21. Novitckii P.V., Zograf I.A. Otsenka pogreshnostey rezultatov izmerenii [Error estimate of measuring results]. Leningrad, Energoizdat Publ., 1991. 304 p.

22. Guva A.Ya. Kratkiy teplofizicheskiy spravochnik [Brief thermophysical reference book]. Novosibirsk, Sibvuzizdat Publ., 2002. $300 \mathrm{p}$.

23. Garcia A.L. Numerical methods for physics. San Jose CA, CreateSpace Independent Publishing Platform, 2015. 432 p.

24. Babichev N.A. Fizicheskie velichiny: spravochnik [Physical quantities: reference book]. Moscow, Energoatomizdat Publ., 1991. $1232 \mathrm{p}$.

Received: 28 November 2018.

\section{Information about the authors}

Viacheslav Yu. Polovnikov, Cand. Sc., associate professor, National Research Tomsk Polytechnic University. 